Research Article

\title{
Study of Structure and Electro-Optical Characteristics of Indium Tin Oxide Thin Films
}

\author{
N. M. Khusayfan ${ }^{1}$ and M. M. El-Nahass ${ }^{2}$ \\ ${ }^{1}$ Physics Department, Sciences Faculty for Girls, King Abdulaziz University, Jeddah 21589, Saudi Arabia \\ ${ }^{2}$ Physics Department, Faculty of Education, Ain Shams University, Roxy, Cairo 11757, Egypt
}

Correspondence should be addressed to N. M. Khusayfan; khusayfan@gmail.com

Received 17 March 2013; Revised 29 May 2013; Accepted 5 June 2013

Academic Editor: Dario Alfe

Copyright (C) 2013 N. M. Khusayfan and M. M. El-Nahass. This is an open access article distributed under the Creative Commons Attribution License, which permits unrestricted use, distribution, and reproduction in any medium, provided the original work is properly cited.

\begin{abstract}
ITO thin films were prepared by electron beam evaporation of ceramic ITO target. The films were subsequently annealed in air atmosphere at the temperatures $300^{\circ} \mathrm{C}$ and $600^{\circ} \mathrm{C}$ in order to improve their optical and electrical properties. The crystal structure and morphology of the films are investigated by X-ray diffraction and scanning electron microscope techniques, respectively. The films exhibited cubic structure with predominant orientation of growth along (222) direction, and the crystallite size increases by rising annealing temperature. Transparency of the films, over the visible light region, is increased by annealing temperature. The resulting increase in the carrier concentration and in the carrier mobility decreases the resistivity of the films due to annealing. The absorption coefficient of the films is calculated and analyzed. The direct allowed optical band gap for as-deposited films is determined as $3.81 \mathrm{eV}$; this value is increased to 3.88 and $4.0 \mathrm{eV}$ as a result of annealing at $300^{\circ} \mathrm{C}$ and $600^{\circ} \mathrm{C}$, respectively. The electrical sheet resistance is significantly decreased by increasing annealing temperature, whereas figure of merit is increased.
\end{abstract}

\section{Introduction}

Conducting oxide thin films are being an important component in different optoelectronic devices such as solar cells [1], light emitting diodes [2], photodiodes [3], and electrochromic devices [4] in which they are used as transparent electrodes. The resistivity of these electrodes should be minimized as much as possible with keeping its high optical transparency particularly over the visible region of the solar spectrum. Indium tin oxide (ITO) thin films are being the most used material, the interest of which has been trusted by fundamental advantages over the competing materials. ITO thin films have been manufactured by using a variety of methods including direct current and radiofrequency sputtering $[5,6]$, reactive evaporation [7], pulsed laser ablation [8], electron beam evaporation [9], spray pyrolysis [10], and sol-gel techniques [11].

Studies on ITO films, grown by the above-mentioned deposition techniques, revealed that the electro-optical properties of such films are highly sensitive towards the method of preparation, composition, partial pressure of oxygen, substrate temperature, substrate type, and annealing processes. The microstructure of the films could be changed with the change of annealing temperature and hence could affect the optical and electron transport properties. Furthermore, the identification of intrinsic changes to ITO characteristics is crucial because these films are eventually used in further applications that involve more treatment processes, which in turn could alter the initial optimized properties. The effect of annealing temperatures on the properties of ITO films deposited onto quartz substrates is a topic that has received little attention from previous studies. Using quartz as substrate for ITO films validity heating such films at higher temperatures allows investigation of their intrinsic properties, as the quartz substrates are transparent in the whole optical range of interest.

Amongst the different techniques available, electron beam evaporation seems to be the most attractive one for preparing ITO thin films. The advantages of this technique can be found in our previous work [12]. In that work, we have investigated the thickness dependence of structural and optical properties of indium tin oxide thin films prepared 
by electron beam evaporation onto quartz substrates. As a continuation of our study, we report herein achieving better optical and electrical characteristics. We have chosen an appropriate film thickness $(\sim 200 \mathrm{~nm})$ to describe the effect of annealing temperatures as a sensitizer tool to improve the optical and electrical properties of the films. The paper is organized as follows. In the following section, the experimental details are given. In Section 3 , the obtained results are presented and discussed. Finally, conclusions are drawn.

\section{Experimental}

Deposition of the ITO thin films was performed by using vacuum coating unit equipped with an electron beam evaporation system. The source evaporation material was ITO pellet with composition of 90 wt. $\%$ of $\mathrm{In}_{2} \mathrm{O}_{3}$ and $10 \mathrm{wt} . \%$ of $\mathrm{SnO}_{2}$ obtained from Aldrich Company. ITO thin films were deposited onto precleaned optically flat quartz substrates and glass substrates with dimensions of $25 \times 25 \mathrm{~mm}$ held at room temperature.

Prior to starting evaporation processes, a steady-state chamber pressure of about $2.6 \times 10^{-3} \mathrm{~Pa}$ (base pressure) was reached. Oxygen (99.99\%) was introduced into the deposition system from a steel tube through a calibrated leak valve. The working pressure during the film deposition was fixed at $1 \times 10^{-2} \mathrm{~Pa}$ by adjusting the needle valve for the ultrapure oxygen gas flow. The deposition rate $(0.2 \mathrm{~nm} / \mathrm{s})$ and the film thickness were controlled and monitored during the evaporation process by using a quartz crystal sensor (Model, TM-350 MAXTEK, Inc., USA). The produced films were thermally annealed at temperatures of 300 and $600^{\circ} \mathrm{C}$ in air atmosphere and at dark condition. The optimized annealing time was one hour. The temperature of the samples was recorded by using $\mathrm{NiCr}-\mathrm{NiAl}$ thermocouple with an accuracy of $\pm 1^{\circ} \mathrm{C}$.

The X-ray diffractometer (Philips $\mathrm{X}^{\prime}$ pert) with Ni-filtered $\mathrm{CuK}_{\alpha}$ radiation $(\lambda=1.5418 \AA)$ was used to identify the structure characteristics of ITO thin film in the scan range of diffraction angles between $5^{\circ}$ and $80^{\circ}$ with a step size of 0.02 $(2 \theta / \mathrm{s})$. The applied voltage and the tube current were $40 \mathrm{kV}$ and $30 \mathrm{~mA}$, respectively. The morphology of ITO thin films was investigated by using a scanning electron microscopy (Model Philips XL 30) with an accelerating voltage of $30 \mathrm{kV}$.

A double-beam spectrophotometer (JASCO model V570 UV-VIS-NIR) was used to measure the transmittance and reflectance of ITO thin films, deposited onto quartz substrates, in the spectral range of 200-2500 $\mathrm{nm}$. The absolute values of the measured transmittance and reflectance after introducing corrections, resulting from the absorption and reflection of the substrate, are determined by the expressions given in [13].

The sheet resistance measurements were carried out at room temperature by using a standard four-probe technique (van der Pauw technique). The electrical electrodes were made of silver films at the corners of the substrate. The silver contacts were tested to be ohmic. Silver films were produced by using a high-vacuum evaporation coating unit (model E 306 A, Edwards Co., England). Prior to deposition, the

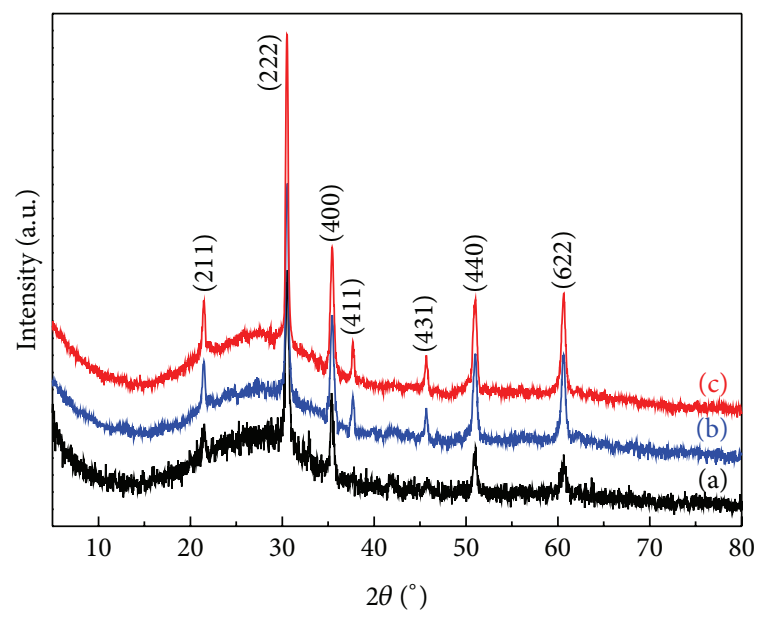

FIGURE 1: X-ray diffraction spectra of ITO thin films with a thickness of $199 \mathrm{~nm}$ : (a) as-deposited, (b) annealed at $300^{\circ} \mathrm{C}$, and (c) annealed at $600^{\circ} \mathrm{C}$.

chamber was evacuated to a pressure of $5 \times 10^{-4} \mathrm{~Pa}$. The experimental details of measuring the sheet resistance can be found in [14].

\section{Results and Discussion}

3.1. Structural Characteristics. X-ray diffraction (XRD) is used to identify the crystal structure of ITO thin films and to analyze the effect of annealing temperature on their optical and electrical properties. Figure 1 depicts the X-ray scattering intensity as a function of diffraction angle for as-deposited and annealed ITO thin film with a thickness of $199 \mathrm{~nm}$. The as-deposited film exhibits well crystalline structure with five diffraction peaks. The reason for obtaining well crystalline films at room temperature is that the kinetic energy from the evaporated particles enhances the surface migration which in turn results in well crystallization. Upon annealing, the initial peaks are still present, and new two diffraction peaks are detected. Moreover, the intensity of the diffraction peaks is obviously increased after annealing, implying the improvement of crystalline structure. Analysis of crystal structure revealed that the patterns can be indexed to $\mathrm{In}_{2} \mathrm{O}_{3}$ with a cubic structure [15]. None of the spectra indicated any characteristic peaks of $\mathrm{Sn}, \mathrm{SnO}$, or $\mathrm{SnO}_{2}$, indicating complete miscibility of $\mathrm{In}$ and $\mathrm{Sn}$ atoms in the $\mathrm{In}_{2} \mathrm{O}_{3}$ lattice [16]. It is well known that in ITO material, $\mathrm{Sn}$ is tetravalent, each Sn (IV) replacing In (III) substitutionally, and thereby, donating free electrons for conductivity in the process. So, the ITO retains the cubic $\mathrm{In}_{2} \mathrm{O}_{3}$ structure up to the solid solubility limit of the $\mathrm{SnO}_{2}$ in $\mathrm{In}_{2} \mathrm{O}_{3}$ [17]. It is worth noting that the increase in the annealing temperature resulted in more orientation along (222) direction, and this preferred orientation is unchanged with increasing annealing temperature up to $600^{\circ} \mathrm{C}$. This behavior is consistent with that observed by Liu et al. [18] for ITO thin films prepared by solgel technique onto quartz substrates in which the films were annealed at temperatures starting from $400^{\circ} \mathrm{C}$ up to $800^{\circ} \mathrm{C}$. 


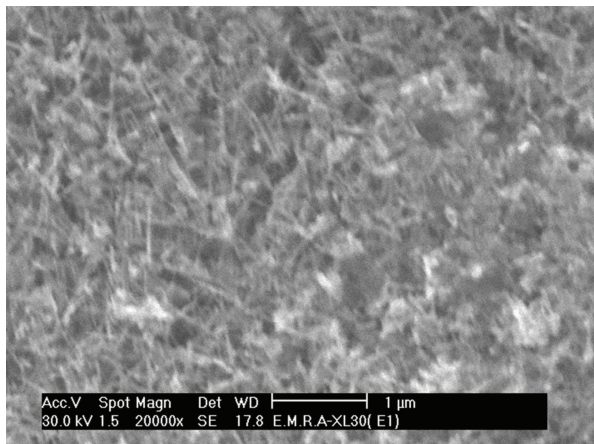

(a)

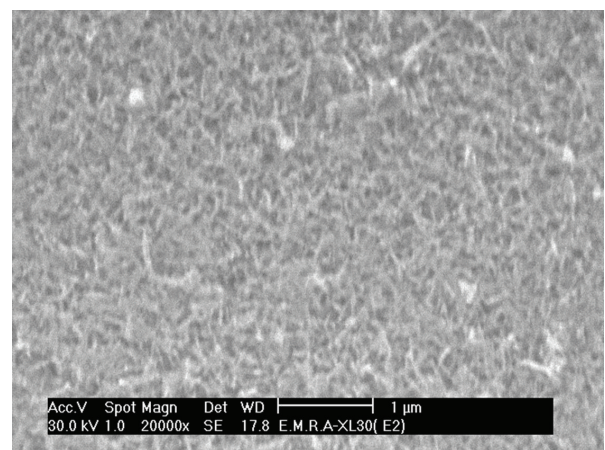

(b)

FIGURE 2: SEM images of ITO thin film with a thickness of $199 \mathrm{~nm}$ : (a) as-deposited, (b) annealed at $600^{\circ} \mathrm{C}$.

The lattice parameter is an important piece of information that can be derived from the X-ray diffraction data. Utilizing the measured interplanar spacing of the main crystal plane (222) for cubic structure, the lattice constant $a_{o}$ of the films is calculated using the following equation [19]:

$$
\frac{1}{d_{h k l}}=\frac{\sqrt{h^{2}+k^{2}+l^{2}}}{a_{o}}
$$

where $d_{h k l}$ is the experimentally measured interplanar spacing (obtained from the XRD peak (222) position) and $h k l$ is corresponding Miller indices. The lattice distortion can be defined as follows [19]:

$$
\left(\frac{\Delta d}{d_{S}}\right)=\frac{d_{h k l}-d_{S}}{d_{S}}
$$

where $d_{S}$ is the interplanar spacing calculated from unstained lattice constant of $10.118 \AA$ for ideal lattice of $\operatorname{In}_{2} \mathrm{O}_{3}$ [20].

An estimation of the crystallite size along a line normal to the preferentially oriented crystal plane $(h k l)$ is possible from $\mathrm{X}$-ray spectra by means of Debye-Scherrer formula [21]:

$$
C S_{h k l}=\frac{0.9 \lambda}{\beta_{h k l} \cos \theta_{h k l}},
$$

where $\lambda$ is the wavelength of X-rays, $\beta_{h k l}$ is the broadening of diffraction line at half of its maximum intensity in radian of the main peak in XRD spectra and $\theta_{h k l}$ is the corresponding Bragg's diffraction angle.

A comparative look of the lattice constant, lattice distortion, and crystallite size of as-deposited and annealed ITO thin films is listed in Table 1. It is observed that higher structural distortion can be related to lower crystallite size. The lattice constant decreases with annealing temperature and approaches the ideal lattice value at $600^{\circ} \mathrm{C}$ [20]. The lattice distortion is also having minimum value after annealing at this temperature which is similar to the reported values for RF-magnetron sputtered films [22]. The improving crystalline structure is demonstrated by the increase in the crystallite size with annealing temperature. The crystallite size increases from 48 to $62 \mathrm{~nm}$ with the annealing temperature and matches well with the reported values $[23,24]$. The
TABLE 1: Structural parameters of as-deposited and annealed ITO thin films.

\begin{tabular}{lccc}
\hline \multirow{2}{*}{ Parameter } & As-deposited & $300^{\circ} \mathrm{C}$ & $600^{\circ} \mathrm{C}$ \\
\hline$a_{o}(\AA)$ & 10.127 & 10.123 & 10.119 \\
$V\left(\AA^{3}\right)$ & 1039.586 & 1037.356 & 1036.127 \\
$\Delta d / d_{o}(\%)$ & 1.19 & 0.92 & 0.72 \\
$C S(\mathrm{~nm})$ & 48 & 53 & 62 \\
\hline
\end{tabular}

systematic increase in crystallite size with increasing annealing temperature is attributed to the sufficient increase in supply of thermal energy for crystallization, recrystallization, and growth of grains in the films and indicates the formation of better crystalline films at higher annealing temperatures.

The surface morphology of the electron beam evaporated ITO thin films was investigated by scanning electron microscope (SEM). Figures 2(a) and 2(b) represent typical SEM images of ITO thin films SEM images of ITO thin film with a thickness of $199 \mathrm{~nm}$ for the as-deposited and annealed films at $600^{\circ} \mathrm{C}$. The overall features of these images indicate that ITO thin films are grown as nanofibers.

Figure 2(b) shows the change in the surface topography after annealing. It is clear that the crystal growth (the length and the width of the rods) is affected by the annealing.

3.2. Electro-Optical Properties. High transparency of conducting oxide thin films, particularly over the visible light region, is a desired property in applications such as transparent electrodes in optoelectronic devices. Owing to that nature, information concerning optical transmittance is an important tool in specifying the optical performance of ITO thin films. Figure 3 demonstrates typical curves of the transmittance and reflectance spectra of as-deposited and annealed ITO thin film with a thickness of $199 \mathrm{~nm}$. The films exhibit high transparency in the spectral range of 400$1300 \mathrm{~nm}$. With the increasing annealing temperature, the transmittance is slightly increased within this spectral range. The results show also a minute increase of the reflectance within the same spectral range as a result of annealing. That the higher annealing temperature resulted in higher optical 


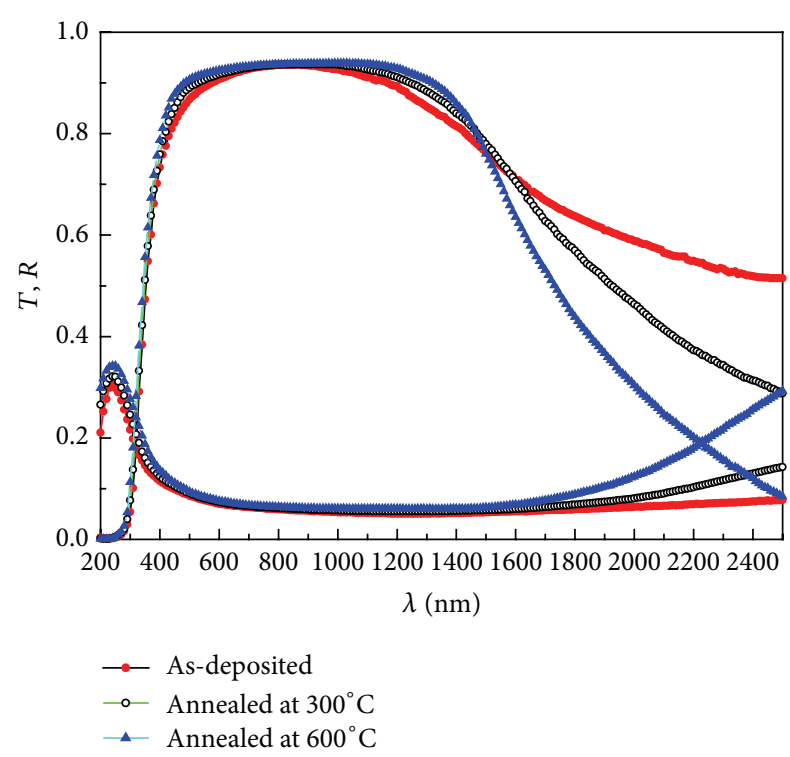

FIGURE 3: Optical transmittance and reflectance spectra of ITO thin film with a thickness of $199 \mathrm{~nm}$.

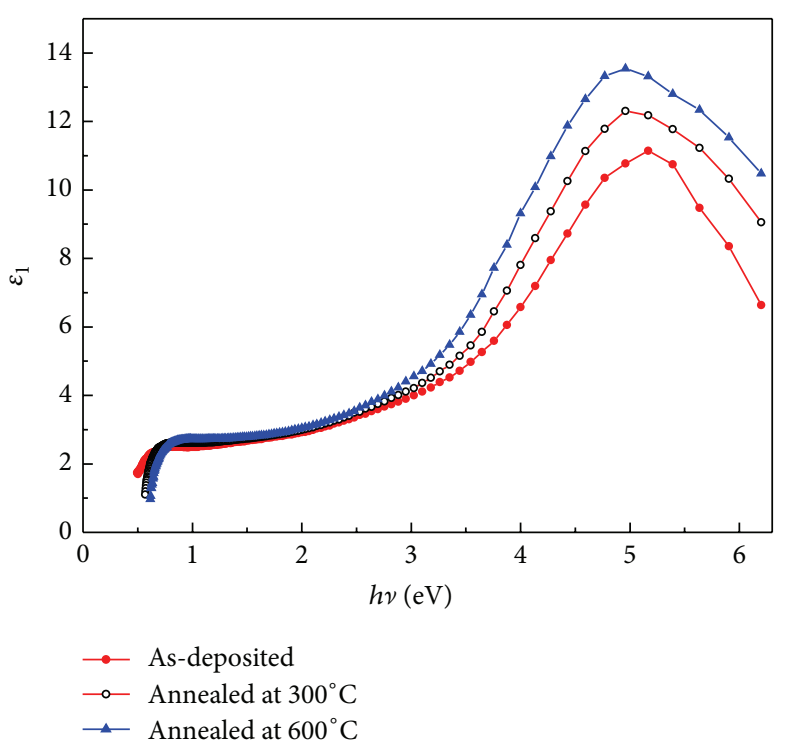

FIGURE 4: Dependence of the real part of dielectric function on the photon energy at different annealing temperatures.

transmittance is attributed to the improvement of structural homogeneity and crystallinity. With the improvement of the crystalline structure, the light wave scattering decreases resulting in better transparency of the films [7]. Over the spectral range of 1300-2500, the transmittance is significantly decreased, whereas the reflectance is increased by annealing. The decrease of the transmittance and the increase of reflectance at high wavelengths occur due to interaction of the incident long-wavelength radiation with the free electrons in the films. The behavior of the transmittance and reflectance for annealed films at $600^{\circ} \mathrm{C}$ is a desirable feature in window layer coatings, especially for application in energy-efficient windows for solar cells, since it can help to prevent unwanted heating. The optical phenomena in this region follow the predictions of classical Drude's model [25]. According to this model, the materials switch from a dielectric to a metallic type behavior due to high free carrier concentration, and this behavior shifts towards shorter wavelengths as the carrier concentration increases. The real part of the complex dielectric function of the films is calculated by [12]

$$
\varepsilon_{1}=n^{2}-k^{2}
$$

where $n$ and $k$ are the refractive and the absorption indices, respectively. The values of $n$ and $k$ are calculated using

$$
\begin{gathered}
n=\frac{1+R}{1-R}+\sqrt{\frac{4 R}{(1-R)^{2}}-k^{2},} \\
k=\frac{\alpha \lambda}{4 \pi},
\end{gathered}
$$

where $\alpha$ is the absorption coefficient given by

$$
\alpha=\frac{1}{d} \ln \left[\frac{(1-R)^{2}}{2 T}+\sqrt{\frac{(1-R)^{4}}{4 T^{2}}+R^{2}}\right],
$$

where $d$ is the film thickness. Figure 4 represents the dependence of the real part, $\varepsilon_{1}$, of the complex dielectric function of ITO films on the photon energy. The plasma energy of asdeposited and annealed films is determined graphically by extrapolating $\varepsilon_{1}$ down to zero value, and the optical carrier concentration, $N_{\text {opt }}$, is calculated by [26]

$$
N_{\mathrm{opt}}=\left(\frac{\varepsilon_{\mathrm{opt}} \varepsilon_{o} m^{*}}{e^{2}}\right) \omega_{p}^{2},
$$

where $\varepsilon_{\mathrm{opt}}$ is the optical dielectric constant, $\varepsilon_{o}$ is the dielectric constant of free space, $m^{*}$ is the effective mass, $e$ is the electronic charge, and $\omega_{p}$ is the plasma frequency. The optical carrier concentration is calculated from (7) by assuming constant value of $m^{*}$ for as-deposited and annealing films, taken as $0.35 m_{o}$ for ITO films [27], where $m_{o}$ is the free electron mass. Besides, the optical dielectric constant was estimated by plotting $\varepsilon_{1}$ versus $\lambda^{2}$ and extrapolating the linear part to $\lambda^{2} \rightarrow 0$.

The resistivity, $\rho$, of the films is determined electrically, and then the mobility, $\mu$, of carriers is calculated by using [26]

$$
\mu=\frac{1}{e N_{\mathrm{opt}} \rho} \text {. }
$$

The carrier concentration, optical dielectric constant, resistivity, and carrier mobility of as-deposited and annealed ITO films are listed in Table 2. Based on the microstructure studies described above and the corresponding optical and electrical parameters measured, a clear correlation between the structural characteristics and electro-optical parameters can be explained. Interestingly, it can be seen that the carrier concentration and carrier mobility are enhanced with 
TABLE 2: Electro-optical parameters of as-deposited and annealed ITO thin films.

\begin{tabular}{lccc}
\hline \multirow{2}{*}{ Parameter } & \multirow{2}{*}{ As-deposited } & \multicolumn{2}{c}{ Annealed } \\
\hline$T($ at $550 \mathrm{~nm})$ & $89.1 \%$ & $90.5 \%$ & $900^{\circ} \mathrm{C}$ \\
$\omega_{p}(\mathrm{rad} / \mathrm{s})$ & $5.51 \times 10^{14}$ & $8.16 \times 10^{14}$ & $9.1 \times 10^{14}$ \\
$N_{\text {opt }}\left(\mathrm{cm}^{-3}\right)$ & $9.67 \times 10^{19}$ & $2.94 \times 10^{20}$ & $4.12 \times 10^{20}$ \\
$\varepsilon_{\text {opt }}$ & 2.89 & 4.00 & 4.51 \\
$\rho(\Omega \cdot \mathrm{cm})$ & $3.03 \times 10^{-3}$ & $4.58 \times 10^{-4}$ & $2.19 \times 10^{-4}$ \\
$\mu\left(\mathrm{cm}^{2} / \mathrm{V} \cdot \mathrm{s}\right)$ & 21.33 & 46.42 & 69.3 \\
$E_{g}(\mathrm{eV})$ & 3.81 & 3.88 & 4 \\
$E_{U}(\mathrm{eV})$ & 0.34 & 0.31 & 0.29 \\
$R_{s}(\Omega / \mathrm{sq})$ & 152 & 23 & 11 \\
$\phi\left(\Omega^{-1}\right)$ & $2.07 \times 10^{-3}$ & $1.6 \times 10^{-2}$ & $3.82 \times 10^{-2}$ \\
\hline
\end{tabular}

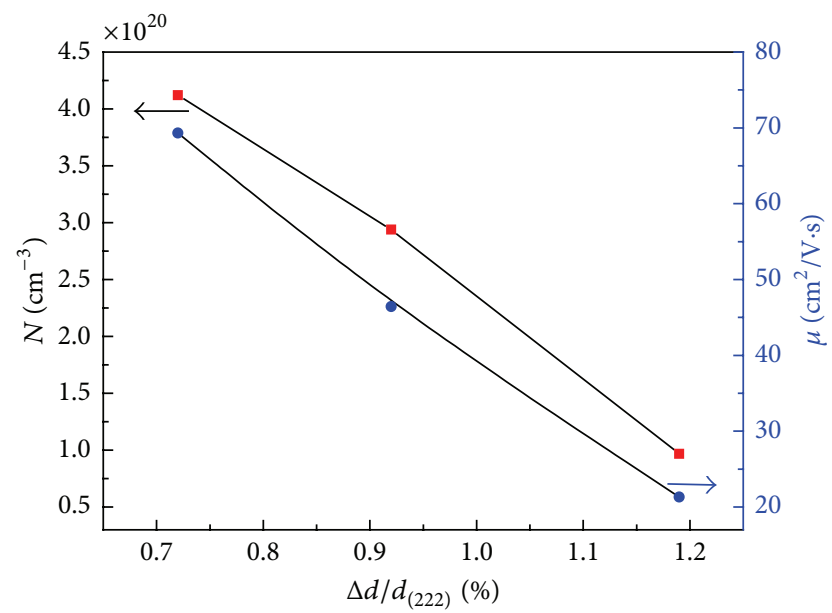

FIGURE 5: Variation of the carrier concentration and carrier mobility with the lattice distortion.

annealing temperature, whereas the resistivity is decreased. This is explained by the fact that free electrons are trapped at the grain boundaries. With increasing grain size, the density of grain boundaries decreases, and fewer carriers are trapped at them, resulting in higher amount of free carriers. Grain boundaries behave also as traps for free carriers and barriers for carriers transport; this explains the increase of carrier mobility with annealing temperature. The values of carrier mobility for the tested samples are in accordance with the published values for ITO films prepared by pulsed laser deposition [28]. Thus, the decrease in resistivity is due to an increase in both the carrier mobility and the carrier density of the films. The magnitudes of electrical resistivity for our samples are in agreement with the reported values $[29,30]$. They correspond to degenerate semiconductors with high free-electron concentration, more than $10^{19} \mathrm{~cm}^{-3}$.

The electro-optical parameters can be correlated to lattice distortion data. Figure 5 illustrates the variation of carrier concentration and carrier mobility with the lattice distortion. It can be observed that free carrier concentration decreases when distortion increases. That is to say, larger distortion

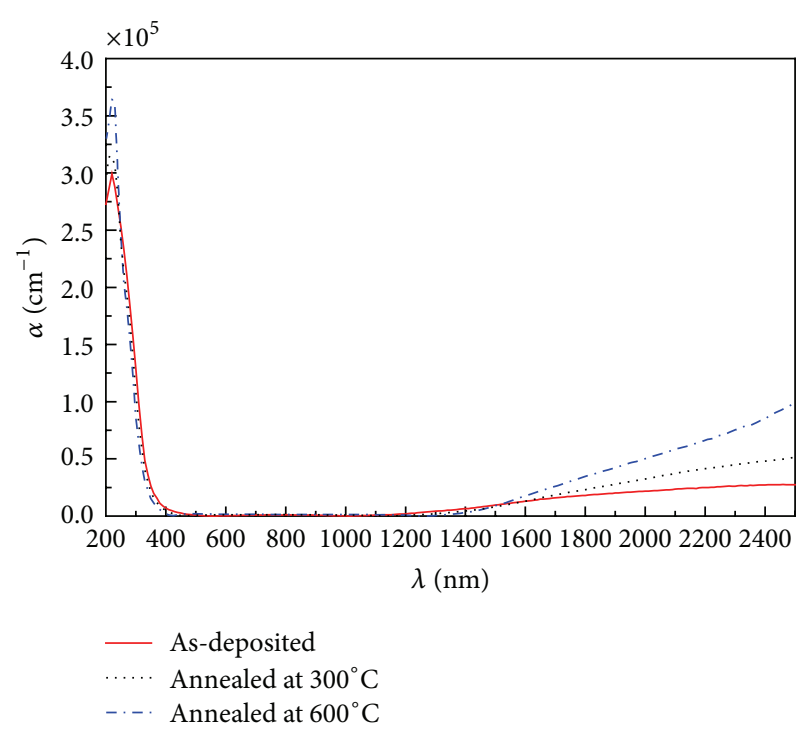

Figure 6: Dependence of the absorption coefficient of ITO thin films on the light wavelength for as-deposited and annealed films.

allows including more oxygen interstitials in the lattice, leading to lower free carrier concentrations by filling the oxygen vacancies or by deactivating the Sn donors. Furthermore, lower distortion allows also the presence of lower defects in lattice structure which enhances the carrier transport resulting in higher mobility.

The influence of annealing temperatures on the absorption coefficient spectra for ITO thin film with a thickness of $199 \mathrm{~nm}$ is demonstrated in Figure 6. The analysis of the absorption coefficient spectra of the films reveals the contribution from different absorption processes. In the ultraviolet region, the absorption is strong due to excitations across the fundamental band gap. The optical properties around fundamental absorption edge are complicated by the occurrence of logarithmic band edges, often referred to as "Urbach tails," which give some weak absorption at the blue end of the luminous spectrum. Urbach tails are common features in semiconductor materials. Usually, the exponential behavior of the absorption coefficient is attributed to the electronic transition in the localized states tailed off in the energy band gap. In the wavelength interval $400-1240 \mathrm{~nm}$, the absorption is very low, that is, transparent region. Finally, in the wavelength interval $1240-2500 \mathrm{~nm}$, the main origin of the absorption is the free carrier absorption. The absorption coefficient, in this wavelength range, increases by increasing annealing due to the increase of free carrier density.

For further analysis, assuming parabolic band scheme of ITO thin films, the type of electronic interband optical transition and optical band gap can be determined from the analysis of the absorption coefficient as a function of the photon energy at the fundamental absorption edge. The optical energy band gap, $E_{g}$, can be estimated using Tauc's method [31]:

$$
\alpha=\frac{A}{h \nu}\left(h v-E_{g}\right)^{s}
$$




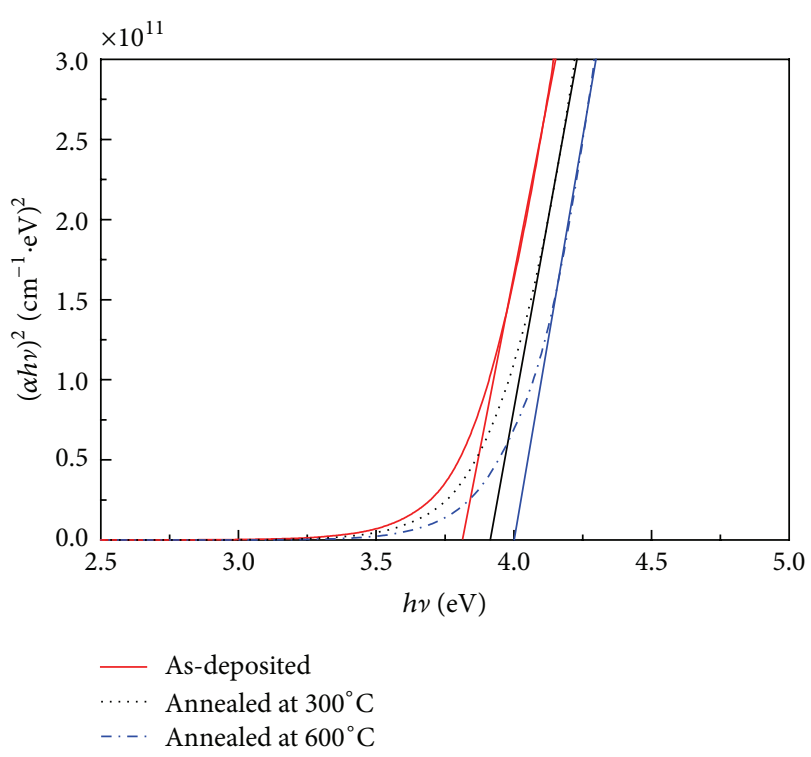

FIGURE 7: Variation of $(\alpha h \nu)^{2}$ with $h v$ for ITO thin films.

where $A$ is a parameter that is proportional to the probability of electron transition between occupied and empty states, $h v$, the photon energy, and the exponent $s$ characterizes the nature of the electronic interband transition; $s=1 / 2$ and $3 / 2$ corresponds to direct allowed and forbidden transitions, respectively, and $s=2$ and 3 corresponds to indirect allowed and forbidden transitions, respectively. The variation of $(\alpha h \nu)^{2}$ with the photon energy for ITO thin films is depicted in Figure 7. The linear portions of the plots near the fundamental band edge in respective figure confirm the direct nature of the band-to-band transitions for respective films. By extrapolating the linear segments of these curves to $(\alpha h \nu)^{2}=0$, the optical band gap values are determined and are given in Table 2 . The obtained optical band gap values correlate well with the published values [32-35] depending on several conditions. It is evident that the optical band gap increases with increasing annealing temperature. This increase is interesting for applications of the films as in window coatings, in order to avoid undesired absorption in the visible spectral range. The optical band gap broadening with increasing annealing temperature can be described in terms of Burstein-Moss (B-M) model [36, 37]. On the bases of this model, the blue shift of the optical band gap is attributed to the filling of states near the bottom of the lowest state in the conduction band due to the increase of the carrier density leading to rise in Fermi level within the conduction band in degenerate semiconductor, which prevents the electronic transition to the lowest levels in conduction band. According to B-M model, the gap energy shift is proportional to the carrier density by the formula:

$$
E_{g}=E_{g 0}+\frac{\hbar^{2}}{2 m^{*} e}\left(3 \pi^{2}\right)^{2 / 3} N^{2 / 3}
$$

where $E_{g 0}$ is the optical band gap of intrinsic $\operatorname{In}_{2} \mathrm{O}_{3}, \hbar$ is Planck's constant divided by $2 \pi$. Figure 8 shows the linear

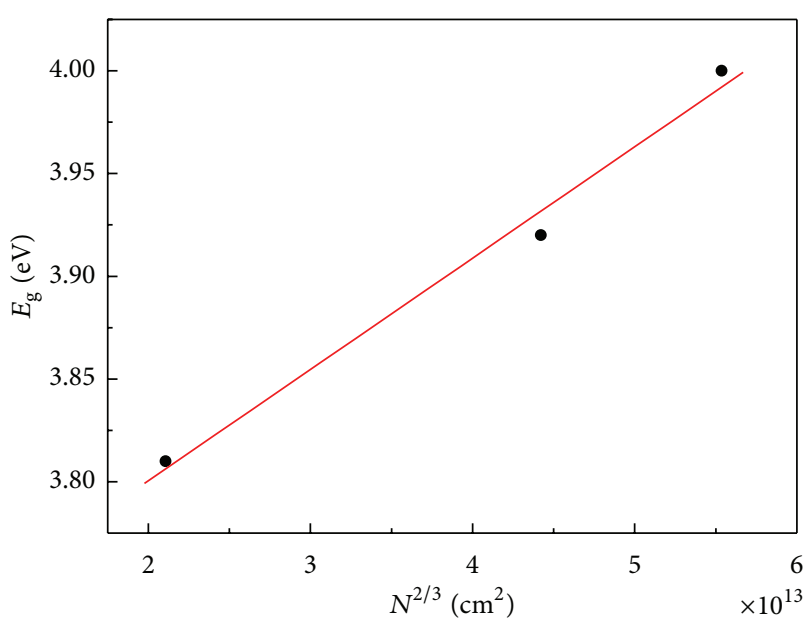

FIGURE 8: The relation between $E_{g}$ and $N^{2 / 3}$ for ITO thin films.

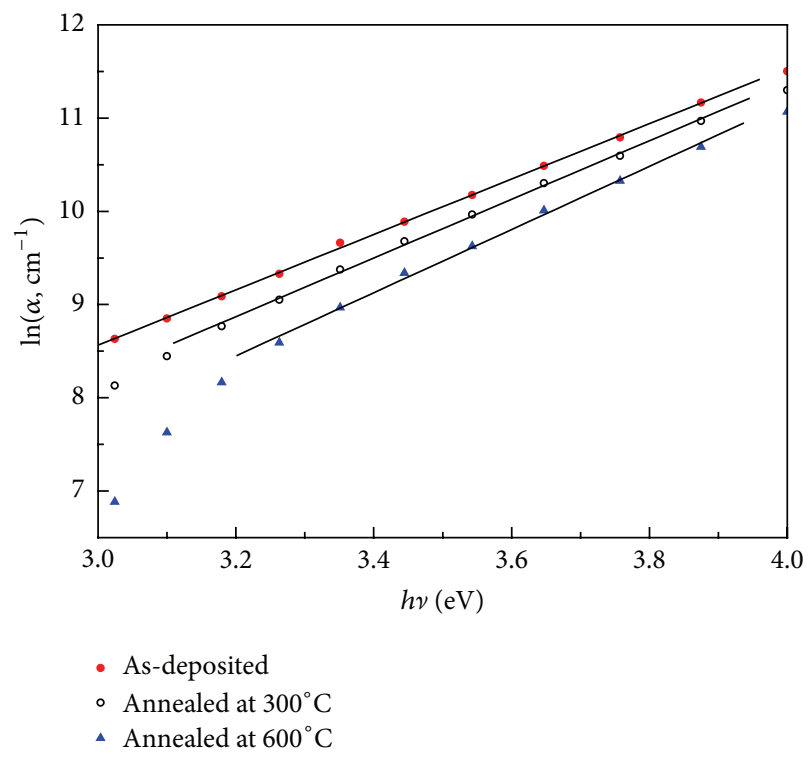

FIGURE 9: Urbach relation for as-deposited and annealed ITO thin films.

variation of the estimated optical band gap versus the carrier density, which is in accordance with the B-M model.

The absorption coefficient tail, directly below the fundamental absorption edge, indicates that there is a high concentration of impurity or defect states causing the band structure to perturb, resulting in a prolonged tail extending into the energy gap of the films. The experimental data are fitted to Urbach's relation [38]:

$$
\alpha=\alpha_{o} \operatorname{Exp}\left(\frac{h v}{E_{U}}\right),
$$

where $\alpha_{o}$ is a characteristic parameter of the material, $E_{U}$ is called Urbach's absorption energy, which can be used as an indicator of the structural defects. The relation between natural logarithm of the absorption coefficient and photon energy is depicted in Figure 9. The relation is linear verifying 
the above equation; the value of $E_{U}$ is estimated for asdeposited and annealed films and is given in Table 2. The value of $E_{U}$ decreases with increasing annealing temperature. This decrease can be attributed to the decrease of residual defects and increase of crystallite size in the films (observed from the XRD studies) which subsequently decreases the density of states in the conduction band tail.

For solar cell application of transparent conducting oxide films, not only high transparency over the visible light region is desired property, but also high conductivity is required. Hence, the figure of merit, defined by Haacke [39], plays an important role for judging the quality of these films. Figure of merit is given by

$$
\phi=\frac{T^{10}}{R_{s}},
$$

where $T$ is the transmittance of the films at specific wavelength (taken at $\lambda=550 \mathrm{~nm}$ [40]) and $R_{s}$ is the electrical sheet resistance. The electrical sheet resistance and figure of merit are given in Table 2. It is evident that sheet resistance decreases continuously with increasing annealing temperature. This is basically due to the increase of the mobility and carrier density at higher annealing temperatures. Obtained sheet resistance value at annealed films at $600^{\circ} \mathrm{C}$ is less than that obtained for electron beam evaporated ITO films onto glass substrates [41]. On the other hand, figure of merit increases with annealing and reaches $3.82 \times 10^{-2}$ for annealed films at $600^{\circ} \mathrm{C}$; this value is consistent with the reported values [42].

\section{Summary and Conclusion}

The evolution of structural, optical, and electrical properties of electron beam evaporated ITO films by thermal annealing temperatures $\left(300\right.$ and $\left.600^{\circ} \mathrm{C}\right)$ is investigated. ITO films deposited at room temperature are found to be in well crystalline structure with a preferential orientation of growth along (222) direction. Subsequent annealing enhances the crystallinity along this direction which results in growth in crystallite size. Lattice constant and lattice distortion decrease with annealing. As-deposited films exhibit high transparency over visible region, annealing increases slightly the optical transparency of the films over this region and improves the reflectance over the near infrared region. The resulting increase in carrier concentration $\left(9.67 \times 10^{19}-4.12 \times\right.$ $\left.10^{20} \mathrm{~cm}^{-3}\right)$ and in carrier mobility $\left(21.33-69.3 \mathrm{~cm}^{2} / \mathrm{V} \cdot \mathrm{s}\right)$ decreases the resistivity of the films $\left(3.03 \times 10^{-3}-2.19 \times\right.$ $\left.10^{-4} \Omega \cdot \mathrm{cm}\right)$ due to annealing. The direct allowed optical band gap is found to increase from $3.81 \mathrm{eV}$ for as-deposited films up to $4 \mathrm{eV}$ for annealed films at $600^{\circ} \mathrm{C}$ which is discussed on the bases of Burstein-Moss model. Urbach's absorption energy is decreased with annealing temperature. Upon annealing, the sheet resistance decreases, and figure of merit increases reaching $11 \Omega / \mathrm{cm}^{2}$ and $3.82 \times 10^{-2} \Omega^{-1}$, respectively, for annealed films at $600^{\circ} \mathrm{C}$. The evolution of electro-optical parameters in this study is related to the improvement in structural characteristics of the films. Eventually, it is realized that the thermal annealing temperature plays a vital role in optimizing the structural and optoelectronic properties of electron beam evaporated ITO films.

\section{Acknowledgment}

This work was funded by the Deanship of Scientific Research (DSR), King Abdulaziz University, Jeddah, under Grant no. (363-009-D1433). The authors, therefore, acknowledge with thanks DSR technical and financial support.

\section{References}

[1] H. M. Zeyada, M. M. El-Nahass, I. K. El-Zawawi, and E. M. El-Menyawy, "Characterization of 2-(2,3-dihydro-1,5dimethyl-3-oxo-2-phenyl-1H-pyrazol-4-ylimino)-2-(4-nitrophenyl)acetonitrile and $\mathrm{ZnO}$ nano-crystallite structure thin films for application in solar cells," The European Physical Journal, vol. 49, p. 10301, 2010.

[2] H. Kim, A. Piqué, J. S. Horwitz et al., "Indium tin oxide thin films for organic light-emitting devices," Applied Physics Letters, vol. 74, no. 23, pp. 3444-3446, 1999.

[3] D. G. Parker and P. G. Say, "Indium tin oxide/GaAs photodiodes for millimetric-wave applications," Electronics Letters, vol. 22, no. 23, pp. 1266-1267, 1986.

[4] J. I. Pankove, Display Devices, Topics in Applied Physics, Springer, Berlin, Germany, 1980.

[5] L. Kerkache, A. Layadi, and A. Mosser, "Effect of oxygen partial pressure on the structural and optical properties of dc sputtered ITO thin films," Journal of Alloys and Compounds, vol. 485, no. 1-2, pp. 46-50, 2009.

[6] G. H. Guai, Q. L. Song, Z. S. Lu, and C.-M. Li, "Effects of multiple heat treatment cycles on structure, optical and electrical properties of indium-tin-oxide thin films," Surface and Coatings Technology, vol. 205, no. 8-9, pp. 2852-2856, 2011.

[7] A. Amaral, P. Brogueira, C. Nunes de Carvalho, and G. Lavareda, "Influence of the initial layers on the optical and electrical properties of ITO films," Optical Materials, vol. 17, no. 1-2, pp. 291-294, 2001.

[8] J. H. Kim, B. D. Ahn, C. H. Lee et al., "Enhancement in electrical and optical properties of indium tin oxide thin films grown using a pulsed laser deposition at room temperature by twostep process," Thin Solid Films, vol. 515, no. 7-8, pp. 3580-3583, 2007.

[9] D. Raoufi, "Morphological characterization of ITO thin films surfaces," Applied Surface Science, vol. 255, no. 6, pp. 3682-3686, 2009.

[10] A. V. Moholkar, S. M. Pawar, K. Y. Rajpure, V. Ganesan, and C. H. Bhosale, "Effect of precursor concentration on the properties of ITO thin films," Journal of Alloys and Compounds, vol. 464, no. 1-2, pp. 387-392, 2008.

[11] H. Y. Valencia, L. C. Moreno, and A. M. Ardila, "Structural, electrical and optical analysis of ITO thin films prepared by solgel," Microelectronics Journal, vol. 39, no. 11, pp. 1356-1357, 2008.

[12] M. M. El-Nahass and E. M. El-Menyawy, "Thickness dependence of structural and optical properties of indium tin oxide nanofiber thin films prepared by electron beam evaporation onto quartz substrates," Materials Science and Engineering B, vol. 177, no. 2, pp. 145-150, 2012.

[13] H. M. Zeyada, M. M. El-Nahass, I. K. El-Zawawi, and E. M. El-Menyawy, "Structural and optical properties of thermally 
evaporated 2-(2,3-dihydro-1,5-dimethyl-3-oxo-2-phenyl-1Hpyrazol-4-ylimino) -2-(4-nitrophenyl)acetonitrile thin films," Journal of Physics and Chemistry of Solids, vol. 71, no. 6, pp. 867-873, 2010.

[14] G. Rietveld, C. V. Koijmans, L. C. A. Henderson et al., "DC conductivity measurements in the Van Der Pauw geometry," IEEE Transactions on Instrumentation and Measurement, vol. 52, no. 2, pp. 449-453, 2003.

[15] Powder Diffraction File, Joint Committee on Powder Diffraction Standards-International Centre for Diffraction Data, Newtown Square, Pa, USA, Card 06-0416, 1981.

[16] P. Sujatha Devi, M. Chatterjee, and D. Ganguli, "Indium tin oxide nano-particles through an emulsion technique," Materials Letters, vol. 55, no. 4, pp. 205-210, 2002.

[17] S. R. Ramanan, "Dip coated ITO thin-films through sol-gel process using metal salts," Thin Solid Films, vol. 389, no. 1-2, pp. 207-212, 2001.

[18] J. Liu, D. Wu, and S. Zeng, "Influence of temperature and layers on the characterization of ITO films," Journal of Materials Processing Technology, vol. 209, no. 8, pp. 3943-3948, 2009.

[19] N. Manavizadeh, F. A. Boroumand, E. Asl-Soleimani et al., "Influence of substrates on the structural and morphological properties of RF sputtered ITO thin films for photovoltaic application," Thin Solid Films, vol. 517, no. 7, pp. 2324-2327, 2009.

[20] L. Hao, X. Diao, H. Xu, B. Gu, and T. Wang, "Thickness dependence of structural, electrical and optical properties of indium tin oxide (ITO) films deposited on PET substrates," Applied Surface Science, vol. 254, no. 11, pp. 3504-3508, 2008.

[21] B. D. Cullity, Elements of X-Ray Diffraction, Addison-Wesley, Reading, Mass, USA, 1956.

[22] L. Kerkache, A. Layadi, E. Dogheche, and D. Rémiens, "Physical properties of RF sputtered ITO thin films and annealing effect," Journal of Physics D, vol. 39, no. 1, pp. 184-189, 2006.

[23] H. R. Fallah, M. Ghasemi, A. Hassanzadeh, and H. Steki, "The effect of deposition rate on electrical, optical and structural properties of tin-doped indium oxide (ITO) films on glass at low substrate temperature," Physica B, vol. 373, no. 2, pp. 274279, 2006.

[24] X. W. Sun, L. D. Wang, and H. S. Kwok, "Improved ITO thin films with a thin $\mathrm{ZnO}$ buffer layer by sputtering," Thin Solid Films, vol. 360, no. 1-2, pp. 75-81, 2000.

[25] P. Drude, "Zur Elektronentheorie der Metalle," Annalen der Physik, vol. 306, pp. 566-613, 1900.

[26] N. M. Torkaman, Y. Ganjkhanlou, M. Kazemzad, H. H. Dabaghi, and M. Keyanpour-Rad, "Crystallographic parameters and electro-optical constants in ITO thin films," Materials Characterization, vol. 61, no. 3, pp. 362-370, 2010.

[27] H. Köstlin, R. Jost, and W. Lems, "Optical and electrical properties of doped $\mathrm{In}_{2} \mathrm{O}_{3}$ films," Physica Status Solidi A, vol. 29, pp. 87-93, 1975.

[28] J. H. Kim, K. A. Jeon, G. H. Kim, and S. Y. Lee, "Electrical, structural, and optical properties of ITO thin films prepared at room temperature by pulsed laser deposition," Applied Surface Science, vol. 252, no. 13, pp. 4834-4837, 2006.

[29] M. Okuya, N. Ito, and K. Shiozaki, "ITO thin films prepared by a microwave heating," Thin Solid Films, vol. 515, no. 24, pp. 86568659, 2007.

[30] L. Lin, F. Lai, Y. Qu, R. Gai, and Z. Huang, "Influence of annealing in $\mathrm{N}_{2}$ on the properties of $\mathrm{In}_{2} \mathrm{O}_{3}: \mathrm{Sn}$ thin films prepared by direct current magnetron sputtering," Materials Science and Engineering B, vol. 138, no. 2, pp. 166-171, 2007.
[31] J. Tauc and A. Menth, "States in the gap," Journal of NonCrystalline Solids, vol. 8-10, pp. 569-585, 1972.

[32] H. Kim, C. M. Gilmore, A. Piqué et al., "Electrical, optical, and structural properties of indium-tin-oxide thin films for organic light-emitting devices," Journal of Applied Physics, vol. 86, no. 11, pp. 6451-6461, 1999.

[33] V. Teixeira, H. N. Cui, L. J. Meng, E. Fortunato, and R. Martins, "Amorphous ITO thin films prepared by DC sputtering for electrochromic applications," Thin Solid Films, vol. 420-421, pp. 70-75, 2002.

[34] S. H. Kim, N.-M. Park, T. Kim, and G. Sung, "Electrical and optical characteristics of ITO films by pulsed laser deposition using a 10 wt. $\% \mathrm{SnO}_{2}$-doped $\mathrm{In}_{2} \mathrm{O}_{3}$ ceramic target," Thin Solid Films, vol. 475, no. 1-2, pp. 262-266, 2005.

[35] C. V. R. Vasnt Kumar and A. Mansingh, "Effect of targetsubstrate distance on the growth and properties of rf-sputtered indium tin oxide films," Journal of Applied Physics, vol. 65, p. 1270, 1989.

[36] E. Burstein, "Anomalous optical absorption limit in InSb," Physical Review, vol. 93, p. 632, 1954.

[37] T. S. Moss, "The interpretation of the properties of indium antimonide," Proceedings of the Physical Society, vol. 67, p. 775, 1954.

[38] F. Urbach, "The long-wavelength edge of photographic sensitivity and of the electronic absorption of solids," Physical Review, vol. 92, p. 1324, 1953.

[39] G. Haacke, "New figure of merit for transparent conductors," Journal of Applied Physics, vol. 147, p. 4086, 1976.

[40] J. H. Chae and D. Kim, "Effect of the $\mathrm{Cu}$ underlayer on the optoelectrical properties of ITO/Cu thin films," Renewable Energy, vol. 35, no. 1, pp. 314-317, 2010.

[41] H. R. Fallah, M. Ghasemi varnamkhasti, and M. J. Vahid, "Substrate temperature effect on transparent heat reflecting nanocrystalline ITO films prepared by electron beam evaporation," Renewable Energy, vol. 35, no. 7, pp. 1527-1530, 2010.

[42] H. R. Fallah, M. Ghasemi, and A. Hassanzadeh, "Influence of heat treatment on structural, electrical, impedance and optical properties of nanocrystalline ITO films grown on glass at room temperature prepared by electron beam evaporation," Physica E, vol. 39, no. 1, pp. 69-74, 2007. 

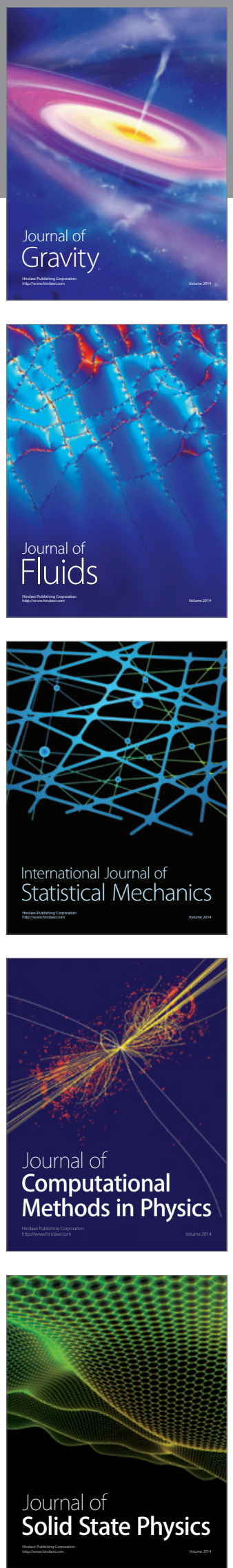

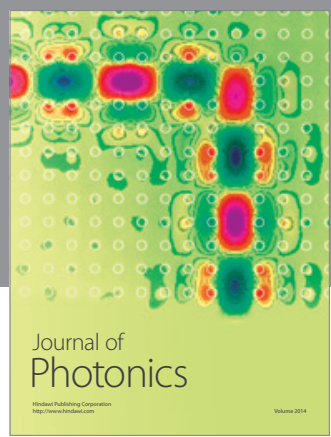

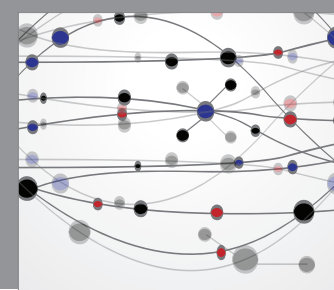

The Scientific World Journal

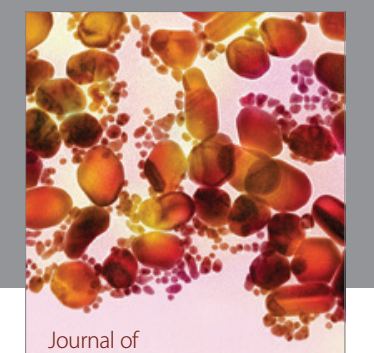

Soft Matter
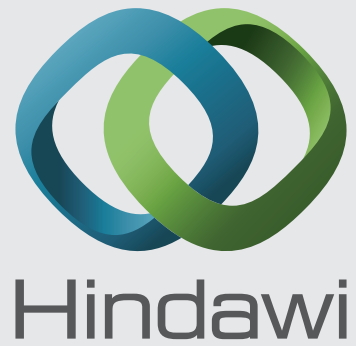

Submit your manuscripts at

http://www.hindawi.com
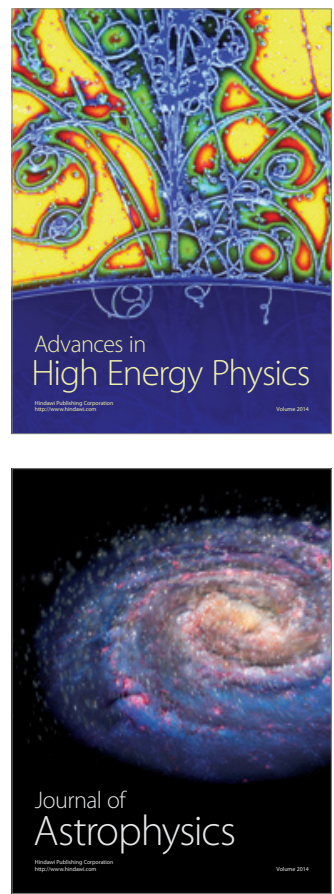
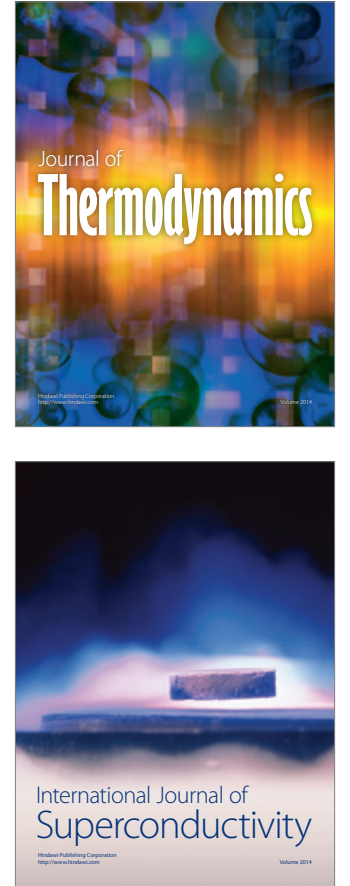
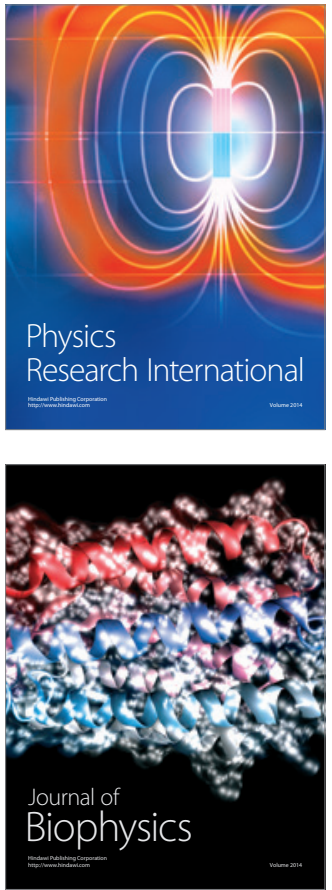
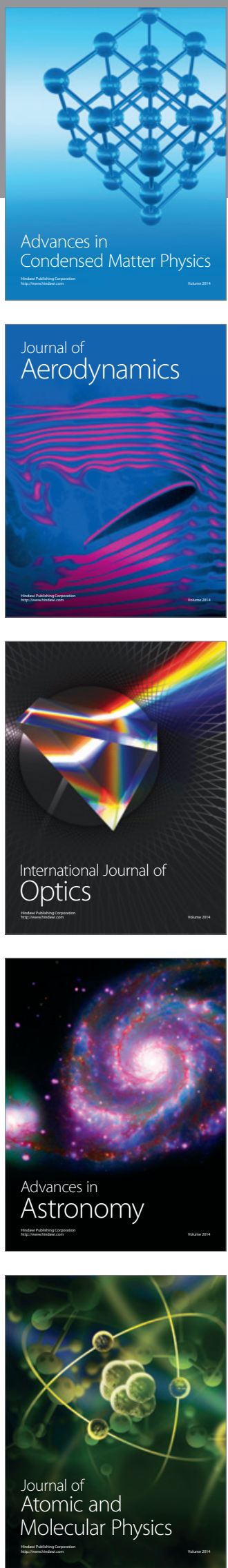